\title{
Sistema de apoyo para la localización de menores de edad desaparecidos utilizando dispositivos móviles
}

\author{
Luis Roberto Conde-Salinas, Jorge Fernando Ambros-Antemate, María del Pilar Beris- \\ tain-Colorado
}

\author{
Escuela de Ciencias, Universidad Autónoma Benito Juárez de Oaxaca, México \\ \{condesitho,jfambros\}@gmail.com, berisp@hotmail.com
}

\begin{abstract}
Resumen. El gran avance tecnológico que han tenido los dispositivos móviles en los últimos años ha permitido un incremento en el número de usuarios, esto presenta un nuevo escenario para el desarrollo de aplicaciones. Hoy en día estos dispositivos son capaces de procesar una mayor cantidad de información, lo que permite utilizar arquitecturas que antes estaban solo disponibles para aplicaciones de computadoras personales. Este artículo propone una aplicación basada en una Arquitectura Orientada a Servicios (SOA, Service-oriented Architecture) utilizando servicios Web, que permita el envío de información de menores de edad desaparecidos a dispositivos móviles para que la ciudadanía ayude en su pronta recuperación.
\end{abstract}

\section{$1 \quad$ Introducción}

Ante la necesidad de brindar mayor protección a los niños, niñas y adolescentes que se encuentren en riesgo inminente de sufrir daño grave a su integridad personal, ya sea por motivo de ausencia, desaparición, extravío, la privación ilegal de la libertad, los gobiernos de varios países en el mundo han implementado medidas de seguridad y difusión para su búsqueda y localización. Es en este sentido, que se requieren nuevas formas para difundir alertas de menores de edad desaparecidos para abarcar una mayor cantidad de personas para que auxilien en la pronta recuperación.

Gracias a que actualmente se ha incrementado el número de dispositivos de comunicación móvil, esto abre un panorama para desarrollar aplicaciones que permitan difundir información a potenciales usuarios, ahora no solo se cuenta con la comunicación por voz, también se tiene la ventaja de enviar y recibir datos gracias a las características que ofrecen las actuales redes en la que se encuentran estos dispositivos, entre las ventajas que ofrecen estos se encuentran [1]: (1) Ubiquidad, es una de las principales ventajas, los usuarios obtiene información en la que están interesados a cualquier hora y en cualquier lugar donde tengan cobertura con acceso a Internet, (2) Accesibilidad, a través de los 
dispositivos móviles los proveedores de servicios tienen un mayor poder de difusión de información, ya que cuentan con los datos de sus clientes, (3) Localización, las aplicaciones desarrolladas con localización ofrecen nuevas posibilidades, al conocer la ubicación de un cliente es posible ofrecer nuevos servicios, como mostrar información en la cual estén interesados.

Para mejorar este tipo de aplicaciones las plataformas de desarrollo juegan un papel importante al adaptar nuevas formas de acceso a datos, como son los servicios Web, los cuales ofrecen una completa interoperabilidad entre las aplicaciones utilizando estándares abiertos, esto permite que el servidor envíe los datos a las aplicaciones móviles clientes y viceversa. Basado en lo anterior, este artículo propone una aplicación que sirva de apoyo para el envío de alertas que ayuden a la localización de menores desaparecidos basada en una Arquitectura Orientada a Servicios (SOA) utilizando servicios Web.

El presente artículo se encuentra estructurado de la siguiente manera: la sección 2 presenta una introducción a la alerta AMBER, utilizada actualmente para difundir información de menores desaparecidos, la sección 3 describe la arquitectura en la cual se basa la aplicación móvil, la sección 4 presenta la implementación de la arquitectura, en la sección 5 se muestra el prototipo de aplicación con un caso de estudio que describe su funcionalidad, la sección 6 presenta trabajos relacionados con la implementación de una SOA en aplicaciones para dispositivos móviles. Finalmente, la sección 7 presenta las conclusiones de este artículo.

\section{Alerta AMBER}

El sistema de alerta AMBER inició en 1996 cuando las emisoras del área de Dallas desarrollaron el concepto de utilizar el Sistema de Alerta de Emergencias (Emergency Alert System, EAS) en conjunto con la policía local para transmitir información rápidamente en casos de secuestro de niños. La palabra AMBER se deriva de America's Missing: Broadcast Emergency Response y fue creada en memoria de Amber Hagerman, una niña de 9 años secuestrada mientras montaba en bicicleta en Arlington, Texas, posteriormente fue encontrada asesinada[2].

El concepto en el cual se basa este programa es sencillo: cuando la policía notifique a los medios de comunicación el secuestro de un niño, el público televidente y radioyente recibirá una notificación a través de avisos emitidos con toda la información que esté disponible para que éste proporcione los ojos y oídos adicionales que aumentan la probabilidad de captura del secuestrador del niño antes de que hayan transcurrido las primeras tres horas críticas [3].

En México a partir del 2 de mayo de 2012 el Gobierno Federal, implementó y puso en funcionamiento el Programa Nacional Alerta AMBER, para la búsqueda y localización de niñas, niños, que se encuentren en riesgo inminente de sufrir daño grave a su integridad personal, con la puesta en marcha de esta plataforma, México se convierte en el décimo 
país a nivel mundial, y el primero en América Latina en adoptar el Programa Alerta AMBER. Para su difusión actualmente se utilizan algunos medios de comunicación, como son la televisión, radio, páginas Web, sin embargo, se requieren otras formas de difusión para abarcar a un mayor número de personas que estén enteradas cuando ocurre un suceso de este tipo, de esta forma, al contar con un más personas notificadas, se incrementa la posibilidad de recuperación de los menores.

\section{Arquitectura orientada a servicios}

SOA establece una arquitectura cuyo objetivo es aumentar la eficiencia, agilidad y productividad de servicios en una empresa, un servicio permite exponer las soluciones y es la unidad fundamental de un diseño orientado a servicios, estos elementos son piezas de software independientes, cada uno de ellos con distintas características y funcionalidades [4].

SOA es una combinación de consumidores y servicios que trabajan en conjunto, es guiada por principios y soporta diversos estándares [5]. A diferencia de los sistemas de información tradicionales los cuales tienen los procesos de negocios incrustados, SOA separa esos procesos y los organiza en módulos individuales, esto permite que estén disponibles además para otras aplicaciones.

El principio clave de una arquitectura SOA es el "proceso de abstracción”, esto permite que la ejecución, administración y los cambios en los procesos sean manejados directamente a nivel de negocios de una forma más fácil [6]. Actualmente los dispositivos móviles han incrementado su poder de procesamiento y memoria, esto ha abierto un panorama para el desarrollo de aplicaciones utilizando una SOA mediante el uso de servicios Web.

Las aplicaciones desarrolladas utilizando una arquitectura orientada a servicios, proveen los siguientes beneficios:

1. Utilizan estándares como XML (eXtensible Markup Language), WSDL (Web Services Description Language) y SOAP (Simple Object Access Protocol), lo anterior asegura la interoperabilidad entre los diferentes servicios y las aplicaciones que interactúan entre sí.

2. Los protocolos de comunicación utilizados por los servicios Web son independientes del sistema operativo, plataforma y lenguajes de programación.

3. Los servicios al ser débilmente acoplados permiten que las aplicaciones que los utilicen sean fácilmente escalables, ya que existe una pequeña dependencia entre estos elementos.

4. Existen una alta reutilización de los servicios Web, al ser elementos individuales, cada uno de ellos está disponible para ser utilizando en otro tipo de aplicaciones.

Tomando las ventajas que ofrece una SOA, en la siguiente sección se presenta la implementación de la arquitectura en el desarrollo de la aplicación móvil. 


\section{$4 \quad$ Arquitectura de la aplicación}

La propuesta es implementar una SOA mediante servicios Web para permitir el intercambio de información, de esta forma, cada capa de la arquitectura provee un servicio que es utilizando por la capa adyacente. La figura 1 muestra los componentes.

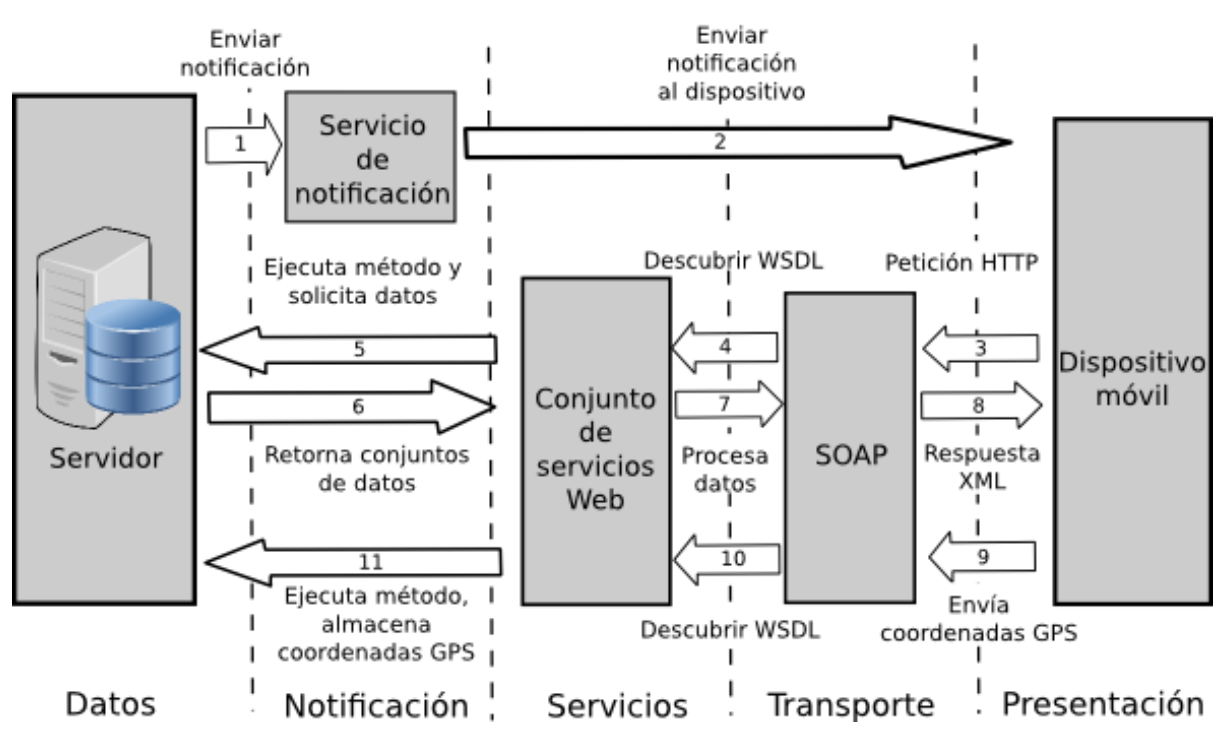

Fig. 1. Arquitectura en capas propuesta para la aplicación.

Las capas y su implementación son descritas a continuación:

Datos: Esta capa es la responsable de almacenar la información referente a los menores reportados como desaparecidos, para la captura de los datos se utiliza una aplicación Web, también almacena los identificadores de los dispositivo que se han registrado, para implementar esta capa se utiliza un servidor MySQL.

Notificación: Se encarga de enviar un mensaje de notificación a los dispositivos que se han registrado en el sistema, la notificación contiene una clave que identifica a un menor desaparecido, para implementar el servicio de notificación se utiliza un servidor externo: Google Cloud Messaging [7][8]. 
Sistema de apoyo para la localización de menores de edad desaparecidos utilizando dispositivos ...

Servicios: Capa responsable de contener los servicios Web, estos son los encargados de realizar todas las transacciones de negocio y exponer las funcionalidades del sistema. Cada servicio Web utiliza estándares para asegurar interoperabilidad entre las aplicaciones: XML como formato estándar para los datos, SOAP es el protocolo utilizado para el intercambio de datos y WSDL para describir las interfaces públicas de los servicios.

Transporte: Se utiliza para administrar los servicios Web mediante el protocolo SOAP, las reglas de negocio están implementadas en el lenguaje de programación PHP, utilizando un grupo de clases específicas para el tratamiento de SOAP.

Presentación: Capa responsable de administrar el flujo de datos hacia el usuario, la aplicación consiste en un cliente ligero que interactúa con el proveedor de servicios, las aplicaciones móviles se diseñan con el patrón Modelo - Vista - Controlador (MVC), donde el Modelo es el encargado de manejar todas las solicitudes realizadas por el Controlador (el responsable de procesar los datos obtenidos de las funciones), finalmente los datos son presentados al usuario en las Vistas.

La funcionalidad de la aplicación se describe a continuación:

Paso 1: Una vez capturada la información del menor desaparecido en el servidor, se envía una notificación al servicio de notificación.

Paso 2: El servicio de notificación envía a uno o varios dispositivos (ya sea por uno o varios estados de la república mexicana) una cadena alfanumérica que representa la clave del menor desaparecido.

Paso 3: Una vez recibida la clave en el dispositivo, se realiza una petición HTTP utilizando la implementación SOAP del cliente, se especifica la URL de la ubicación del documento WSDL.

Paso 4: El cliente SOAP descubre en el servidor el documento WSDL que contiene el método solicitado por la aplicación.

Paso 5: El servidor recibe la petición mediante el protocolo SOAP, la lógica del negocio procesa la solicitud y ejecuta el método correspondiente.

Paso 6: El servidor de base de datos recibe la petición, la procesa y retorna los datos al método que lo solicitó.

Paso 7: Los datos obtenidos del servidor de base de datos son procesados e implementados en el método de la lógica de negocios y enviados mediante el protocolo SOAP.

Paso 8: El cliente SOAP obtiene los datos del menor desaparecido mediante un documento XML, se extrae el contenido para que sea visualizado en el dispositivo móvil.

Paso 9: En caso de tener información de la ubicación del menor desaparecido, se obtienen las coordenadas GPS del dispositivo móvil y se envían por medio del protocolo SOAP al servidor.

Paso 10: Se descubre en el servidor el documento WSDL mediante su URL.

Paso 11: Se ejecuta el método y almacenan las coordenadas GPS, en el servidor se emite una notificación con la información recibida para mostrar en el mapa la ubicación recibida por el usuario.

Otras características de la aplicación son las siguientes: 
- Permite consultar las notificaciones históricas (solo de los menores que no se han localizado aún).

- En caso de no contar con una conexión a Internet la aplicación almacena de manera local en una base de datos la información de los menores, esto permite que los usuarios consulten los datos sin necesidad de contar con una conexión activa.

- $\quad$ Si se tiene información del menor y no se tiene conexión a Internet, el usuario tiene la opción de realizar una llamada telefónica, enviar mensaje de texto o almacenar de manera interna las coordenadas, para que estas últimas sean enviadas al servidor cuando se cuente con el acceso a Internet.

En la siguiente sección se presenta un caso de estudio, donde se muestra la funcionalidad de la aplicación.

\section{$5 \quad$ Caso de estudio}

El siguiente caso de estudio muestra un prototipo de la aplicación móvil desarrollada en la plataforma Android, la aplicación recibe la notificación de alerta, se revisa en el dispositivo móvil los datos del menor, si el usuario obtiene información del menor desaparecido, envía la posición al servidor para mostrar la ubicación mediante un marcador en un mapa.

El proceso inicia cuando el servidor envía una notificación a los clientes registrados en el sistema mediante el servidor externo Google Cloud Messaging, la notificación contiene una clave que identifica los datos del menor desaparecido, la figura 2 (a) muestra la pantalla, esta notificación se realiza por uno o varios estados de la república mexicana, al momento de abrir la notificación en el dispositivo, el cliente SOAP realiza una petición HTTP mediante la URL del servidor que contiene la dirección del documento WSDL, se establece la comunicación con la lógica del negocio y se ejecuta el método solicitado, se obtienen los datos de la base de datos y se construye la respuesta la cual es enviada al cliente utilizando un servicio Web mediante una representación en XML.

Finalmente el cliente obtiene los datos, los separa y los muestra en las vistas, la figura 2 (b) muestra el resultado, donde se presenta la información detallada del menor.

Si el cliente obtiene información del menor desaparecido, dentro de la aplicación tiene la opción de establecer una llamada, enviar mensaje de texto o una ubicación más específica, para esto último se obtienen las coordenadas GPS del dispositivo y son enviadas al servidor, la figura 3 (a) muestra esta pantalla.

Recibidas las coordenadas en el servidor, estas se almacenan en la base de datos y se presenta en el mapa un marcador con la dirección aproximada donde el usuario obtuvo información del menor desaparecido, la figura 3 (b) presenta la pantalla con el mapa y el marcador. 
La aplicación ofrece una nueva forma de apoyar en la localización de un menor, ya que los clientes móviles al recibir notificaciones obtienen información importante, además se tiene la opción de notificar de diferentes maneras a las instancias correspondientes para la pronta recuperación del menor desaparecido.

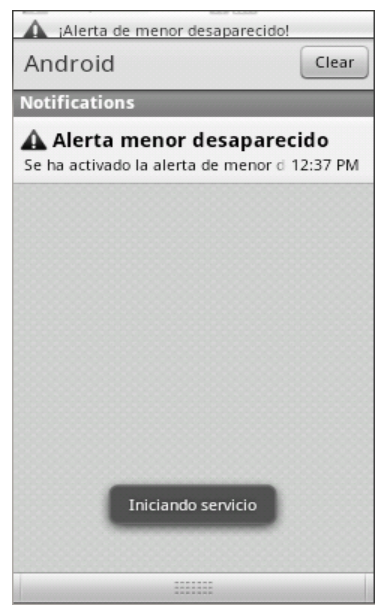

(a)

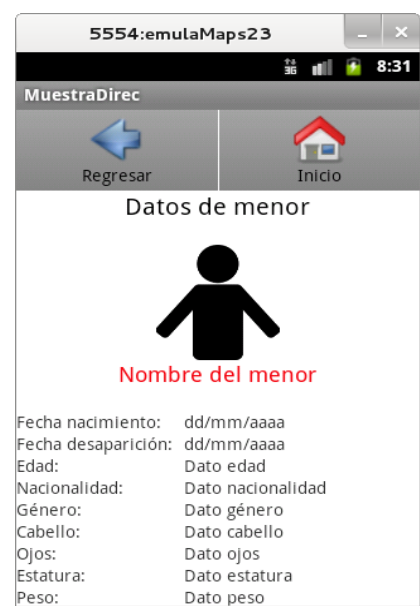

(b)

Fig. 2. (a) Notificación en el dispositivo móvil, (b) pantalla con información del menor desaparecido.

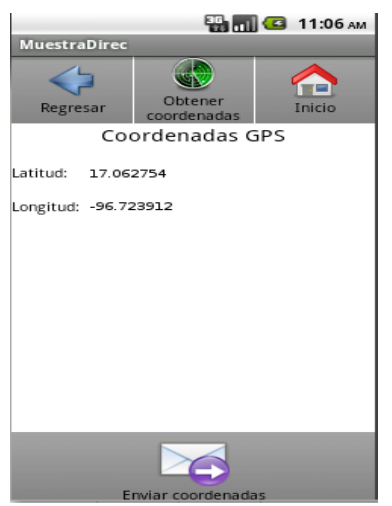

(a)

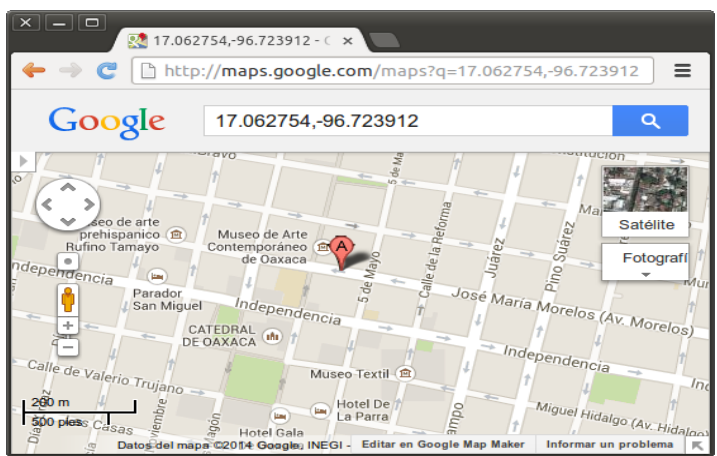

(b)

Fig. 3. (a) Obtención de las coordenadas donde se obtuvo información del menor desparecido, (b) mapa en el servidor que muestra las coordenadas GPS. 


\section{Trabajos relacionados}

A continuación se presentan trabajos relacionados con el desarrollo de aplicaciones para dispositivos móviles utilizando una SOA. En [9] se presenta un artículo de una aplicación basada en contexto de situaciones de riesgo, dependiendo del tipo de situación que se presenta, se envían los datos a los departamentos de emergencia correspondientes, estos últimos envían la ayuda con el equipo que se requiere para el problema que se presenta, para el envío de información a los departamentos de emergencia se utilizan servicios Web. En [10] se realiza un análisis de una arquitectura basada en servicios Web para dispositivos móviles, utilizando J2ME Web Services API para permitir el uso del protocolo SOAP y acceder a los servicios Web.

En [11] se desarrolla una aplicación basada en una SOA, servicios Web y posicionamiento para ofrecer a los turistas información del sitio donde se encuentran, utiliza también realidad aumentada e información adicional proporcionada por otros proveedores de servicios. En [12] se presenta la propuesta de una SOA para una aplicación móvil que permite la recuperación de datos de información criminal, para esto el sistema accede a la información de la escena del crimen desde múltiples servidores, la información es presentada en el dispositivo móvil mediante texto, gráficos, multimedia y características del lugar, el sistema permite también el intercambio de información con los servidores de diversas instituciones de seguridad.

\section{Conclusiones}

Este artículo presenta una aplicación basada en una Arquitectura Orientada a Servicios, la cual provee otra forma de difusión de alertas de menores desaparecidos a usuarios de dispositivos móviles, todo el envío y recepción de información se realiza mediante la invocación de servicios Web utilizando el protocolo SOAP, la implementación del servidor y del cliente se realizó utilizando bibliotecas de funciones de libre distribución, como las clases de manejo SOAP, el lenguaje de programación PHP, en el caso del cliente móvil desarrollado se utiliza la biblioteca de funciones ksoap.

Con el desarrollo de este tipo de aplicaciones utilizando las tecnologías anteriores y gracias al incremento de usuarios con dispositivos móviles en todo el país, se espera abarcar un mayor número de personas que ayuden a la pronta recuperación de un menor desaparecido, en el momento en que se active la alerta, todos los dispositivos registrados recibirán una notificación para que las personas se conviertan en una extensión más de las autoridades, ya que al contar con ayuda por parte de la ciudadanía se incrementa el número de actores involucrados en este tipo de programas, por lo que también se aumentan las 
posibilidades de encontrar a los menores para salvaguardar su integridad y retornarlos a sus familiares.

\section{Referencias}

1. Lim E. Siau K.: Advances Mobile Commerce Technologies. United States of America: Idea Group Publishing. (2003)

2. AMBER alert: About AMBER Alert. [en Web]. Disponible en: http://www.amberalert.gov/about.htm. Date of consultation: (mayo 2014)

3. La Alerta AMBER - Guía de las mejores prácticas para radiodifusores y otros medios de comunicación (Spanish Version). [on Web]. Available: https://www.ncjrs.gov/html/ojjdp/amberalert/209519/pg2.html

4. Erl Thomas: SOA, Principles of Service Design: Prentice Hall. (2007)

5. Bean J. SOA and Web Services Interface Design. United States of America: Elsevier Inc. (2010)

6. Fernández J., Surroca A.: Cómo reformular la Arquitectura Corporativa para alcanzar el alto Rendimiento. Centro de Alto rendimiento: (2008)

7. Wang Ke-feng: The Design and Implementation of Information Push and Management System Based on Android, (2012)

8. Penghui Li: Implementation of Cloud Messaging System Based on GCM Service. Computational and Information Sciences (ICCIS), 2013 Fifth International Conference. (2013)

9. Romsaiyud, W., Premchaiswadi, W.: SOA context-aware mobile data model for emergency situation. Knowledge Engineering, 2010 8th International Conference on ICT.

10. Tergujeff T., Haajanen J., Leppänen J., Toivonen R.: Mobile SOA: Service Orientation on Lightweight Mobile Devices. 2007 IEEE International Conference on Web Services. (2007).

11. Paganelli F., Parlanti D., Francini N., Giuli N.: A SOA-Based Mobile Guide to Augment Tourists' Experiences with User-Generated Content and Third-Party Services, iciw, pp.435-442, 2009 Fourth International Conference on Internet and Web Applications and Services, (2009)

12. Khemprasit, J., Esichaikul, V.: SOA-based framework for mobile crime analysis and monitoring, Electrical Engineering/Electronics, Computer, Telecommunications and Information Technology (ECTI-CON), (2014) 